\title{
A NOTE ON TRANSLATIONS AND ORTHOGRAPHY
}

\section{๙ै।}

Unless otherwise noted, translations are mine.

A note on orthography: Double quotation marks are used for exact quotations and as scare quotes. Italics are used throughout to draw attention to terms as terms as well as for foreign terms and for special emphasis. In block quotes, Greek lettering is preserved; when quoting in the text, Greek words and phrases are transliterated into Roman script. 

ODYSSEYS OF RECOGNITION 
